\title{
EL TURISMO COMO EJE DE ACUMULACIÓN
}

\author{
Alejandro Palafox Muñoz ${ }^{1}$ \\ Universidad de Quintana Roo, México
}

http://dx.doi.org/10.5209/rev_NOMA.2013.42347

\begin{abstract}
Resumen.- La actividad turística es reconocida por su capacidad en la generación de ingresos económicos y empleos, pero también por los impactos negativos resultado de los cambios ocasionados en el paisaje natural y cultural de las localidades. En este sentido, en el marco del modo de producción capitalista, el turismo se ha constituido en un instrumento para la reproducción del capital, a través de su expansión en las economías subdesarrolladas, las cuales concentran una diversidad natural y cultural que la actividad necesita para motivar el desplazamiento. El Estado, juega un rol importante en la consolidación de la actividad turística, ya que éste se apropia del territorio para transformarlo y adecuarlo a las necesidades de los Grupos Turísticos Internacionales (GTI), los cuales mediante la globalización económica han logrado instaurarse en zonas ricas en recursos naturales y culturales, mismas que son modificadas para crear atractivos turísticos determinando así, la nueva vocación del lugar. Aunado a lo anterior, las estrategias utilizadas por los GTI, han sido replicadas en el ámbito local constituyendo los Grupos Turísticos Locales (GTL), en este sentido el estado de Quintana Roo contribuye con el 34\% de los ingresos económicos por concepto de turismo internacional. Por lo anterior, este trabajo pretende revelar el comportamiento de los GTI y los GTL en la entidad y sustentar de esta manera el nuevo rol de la actividad turística: como eje de acumulación.
\end{abstract}

Palabras clave.- turismo; eje de acumulación; transformación del paisaje; apropiación

\section{Tourism as a axis of accumulation}

Abstract.- Tourist activity is recognized for its ability to generate income and jobs, but also by the negative result of the changes caused in the natural landscape and cultural locations. Here, under the capitalist mode of production, tourism has become an instrument for the reproduction of capital, through its expansion in underdeveloped economies, which have natural and cultural diversity that the activity needs to encourage mobility. The state plays an important role in the consolidation of tourism, as it takes over the territory to transform and adapt to the needs of International Tourism Group (IWG), which by economic globalization have been instituted in Rich areas with natural and cultural resources, these are modified to create tourist attractions in consecuence determining the new vocation of the place. Added to this, the strategies used by the IWG have been replicated at the local level constituted the Local Tourist Groups (LTG), in this sense the state of Quintana Roo contributes to $34 \%$ of income from tourism international. Therefore, this paper aims to reveal the behavior of the GTI and GTL in the state and thus support the new role of tourism: the axis of accumulation.

Keywords.- tourism; axis of accumulation; landscape transformation; appropiation

\footnotetext{
${ }^{1}$ Profesor - Investigador de Tiempo Completo. Universidad de Quintana Roo. División de Desarrollo Sustentable. Ave. 11 esquina 110 Sur, Col San Gervasio, Cozumel, Quintana Roo, México. C.P. 77660. Tel + 52 (987) 872 9000. E mail: alejandro.palafox@gmail.com
} 


\section{Introducción}

El turismo moderno es alumno del consumo y de la globalización (Hillali; 2003), su estructura en el ámbito global deviene de los intereses de los organismos internacionales por reproducir el modo de producción capitalista. Por su parte, las economías con un desarrollo incipiente presentan las condiciones ideales para su apropiación, homogenización y funcionalización, es decir, cuentan con recursos naturales prístinos y culturales de interés para otros, mismos que motivan el desplazamiento de los visitantes y el interés de las empresas para invertir, dada la escasez de infraestructura de acceso y servicios que se enfatiza en la región Latinoamericana y del Caribe, sobre todo en los espacios rurales. De esta manera, la políticas de orden liberal reflejan un mínimo involucramiento del Estado en la economía, lo que deriva en altos niveles inequidad social y económica (Webster, Ivanov e Illum; 2009).

El paisaje integrado por los recursos naturales y culturales son de interés para la economía global al ser considerados la materia prima para la planeación, organización, desarrollo y comercialización del turismo, aunado a la necesidad del modo de producción capitalista por transformarse y revitalizarse. Para ello, los organismos internacionales reconocen que el turismo es un medio para la consolidación del modelo cultural vinculado al modelo económico, así la generación de divisas, el impulso a la inversión privada y crear un nuevo producto de exportación sin la necesidad de costos de producción, aunado a la necesidad de los Estados subdesarrollados por la creación de empleos y combatir la pobreza, hacen que la actividad turística se constituya en un eje de acumulación.

Un actor social importante en la reproducción del modo de producción es el Estado, quien juega un papel importante en la consolidación del nuevo eje de acumulación, al dotar de las condiciones estructurales para la llegada de capitales extranjeros en el territorio donde se encuentran los recursos naturales y culturales, mismos que pertenecen a las comunidades. Tales acciones de promoción de la inversión han provocado el fortalecimiento y expansión de las empresas que se han establecido en los países capitalistas emergentes, lo cual dio lugar a la construcción de encadenamientos productivos tales como los Grupos Turísticos Internacionales [GTI] (Cfr. Palafox, Zizumbo y Arriaga; 2010), los cuales son fundamentalmente empresas hoteleras que con ayuda de los procesos de globalización y modernización económica se han diversificado y por tanto participan en diversos mercados no sólo del ámbito turístico. Dicha estrategia de encadenamiento productivo se ve reflejada también en los destinos de corte tradicional o aquellos que no han sido del todo promovidos por el Estado, en donde el poder local en conjunto con el poder político han creado los Grupos de Turísticos Locales (GTL). 


\section{La internacionalización del turismo}

El desarrollo de la actividad turística se fortalece mediante la inclusión de la clase media a la práctica del turismo a partir de la década de los setenta gracias a la alza en el poder adquisitivo y la amplitud de los horarios fuera de trabajo de los asalariados (Duterme; 2007), sin embargo, por la necesidad del modo de producción capitalista por transformarse y continuar con su reproducción, para ello se han creado nuevas formas para su práctica, lo que derivó en una segmentación de los gustos y preferencias, así como en la creación de productos y servicios.

Por ello, en la década de los setenta aparece el turismo social, en los noventa el ecoturismo y sus vertientes alternativas, todo ello en respuesta y adaptación a las necesidades de los organismos internacionales sobre permear el por demás prostituido desarrollo sustentable. En este sentido, la actividad turística se ha expandido exponencialmente a raíz del ejercicio económico del libre mercado, lo cual ha permitido el aumento en los flujos comerciales y la movilización de consumidores como resultado de las transformaciones tecnológicas, así mismo se coloca como "uno de los pilares más poderosos de la globalización" (Lanfant; 2004 en Duterme: 2007: 9).

En los últimos años, el turismo se ha convertido en el motor económico de los países industrializados y subdesarrollados, debido a su incidencia en el crecimiento y desarrollo económico, toda vez que la actividad genera empleo e ingreso (Stanislav y Webster; 2006). De esta manera, desde la década de los noventa comenzó el impulso al desarrollo de la actividad turística en los Estados económicamente emergentes de América (Ver Tabla 1). En este proceso, el Estado estableció las condiciones necesarias para que las empresas trasnacionales se apropien del territorio, del mercado, a fin de posicionar las empresas prestadoras de servicios, y para ello era necesaria la infraestructura que los Organismos Internacionales financiaban a través de empréstitos, ejemplo de ello, son los 25 millones de dólares que el Banco Interamericano de Desarrollo le otorgó al Banco de México en la década de los setenta para la construcción del Centro Integralmente Planeado de Cancún (INFRATUR; 1970).

Tabla 1. Llegadas internacionales por región (millones de turistas).

\begin{tabular}{|cccccc|}
\hline Región & 1990 & 1995 & 2000 & 2005 & 2010 \\
\hline El Caribe & 11.4 & 14.0 & 17.1 & 18.8 & 19.6 \\
\hline América Central & 1.9 & 2.6 & 4.3 & 6.3 & 7.6 \\
\hline América del Sur & 7.7 & 11.7 & 16.0 & 20.9 & 28.3 \\
\hline \multicolumn{5}{c}{ Fuente: OMT (2012). }
\end{tabular}

No sólo la parte Centro - Sur del continente americano ha crecido en el número de llegadas de turistas internacionales, del mismo modo, en África se ha tenido un aumento del $6.3 \%$ en los últimos cinco años, alcanzando los 50.2 millones de visitantes extranjeros; así mismo el continente Asiático ha incrementado el número 
de arribos en un 5\% durante el mismo periodo, alcanzando la cifra de 217 millones de turistas en el 2011. A pesar de lo anterior, las economías capitalistas absorben el $53.2 \%$ de los mercado internacional y los países en vías de desarrollo el $46.8 \%$ del mercado.

Por su parte México captó 23.4 millones de turistas, mismos que dejaron una derrama económica de 11.86 millones de dólares. Estos datos permitieron que México se posicionara como el décimo país con mayor captación de turistas extranjeros, cifra que no le permite figurar dentro de los primeros diez sitios de mayor derrama económica, el décimo lugar lo ocupa Hong Kong con 24.7 millones de dólares (OMT; 2012).

Lo anterior, ha llevado a afirmar que el turismo se ha convertido en un mecanismo que el Estado utiliza para generar un crecimiento económico, además, "el sector turismo está estrechamente ligado a la fuerza globalizadora que persigue fines de lucro a través de la justicia social" (Reid; 2003 en Zhao y Li; 2006: 203). Así, el turismo se ha posicionado como un referente para la dependencia $y$ codependencia económica de los países capitalistas desarrollados y las entidades emergentes, este comportamiento se ve reflejado en el área de los servicios, en donde el "turismo y los servicios relacionados están fuertemente dominados por los países del norte" (Williams; 2002), en este sentido, y de acuerdo con los datos de la Organización Mundial del Turismo (OMT; 2012) los Estados Unidos de Norteamérica es el segundo lugar en la recepción de turistas internacionales (79.5 millones en 2011) y primero en captación de divisas (116.3 billones de dólares en 2011).

El subdesarrollo de los Estados emergentes ha sido provocado por su integración en la periferia de las economías capitalistas, ya que el intercambio inequitativo entre el centro y la periferia con el sistema es inherente (Mosedale; 2009). En este tenor, el crecimiento de los países subdesarrollados depende de la demanda de bienes y servicios de los Estados desarrollados, por lo que los desequilibrios de éstas impactan directamente en la periferia. Por ello, "el sistema capitalista internacional, nacional y local genera un desarrollo económico para las minorías y un subdesarrollo para las mayorías" (Frank; 1969: 7 - 8), ya que "los países industrialmente más desarrollados no hacen más que poner delante de los países menos progresivos el espejo de su propio porvenir" (Marx; 1999: XIV). En suma a lo anterior, la acumulación de capital de las economías desarrolladas depende de las explotación de los Estados emergentes al basar su crecimiento económico en las ganancias generadas en los anteriores y sobre todo en la generación de la dependencia (Bianchi; 2009).

Por ello, Britton (1991) afirma que la actividad turística "es un vehículo para la acumulación de capital" (p. 451), para alcanzar lo anterior, el Estado a través de una política económica neoliberal y la globalización económica como estrategia asegura las condiciones organizacionales y estructurales para que la inversión de las empresas trasnacionales, a través de los canales de distribución y la conformación de grupos y mercados (Clancy; 1998, Mosedale; 2006, Palafox, 
Zizumbo y Arriaga; 2010; Buades; 2011), lo anterior con el propósito de fortalecer el modo de producción capitalista y ello enfatizar la concentración y centralización del capital.

Sin embargo "el monopolio del capital se convierte en traba del modo de producción capitalista... ese modo de producción trae al mundo los medios materiales de su propia aniquilación" (Marx; 2010: 362 - 363). En este sentido, un resultado invariable del capitalismo es la concentración del poder corporativo y el deterioro de los pequeños negocios quienes están destinados a cerrar o vender, aunado a la imposición de las fuerzas productivas a: a) largas jornadas laborales; b) reducción de salarios; c) inseguridad laboral y contractual; y d) desempleo (Brennan; 2003), este comportamiento es particular de las empresas relacionadas con la actividad turística y sobre todo de aquellas dedicadas a la hospitalidad (Bianchi; 2009).

\section{El proceso de acumulación}

Con el libre mercado, las empresas se especializaron y diversificaron, así mismo conformaron los Grupos Turísticos Internacionales (GTI's) quienes de acuerdo con Clancy (1998) operan en red a través de las Global Commodity Chains (GCC) y no sólo ofertan servicios de hospedaje sino incursionan en la gastronomía, la consultoría, la transportación, la organización de viajes, entre otros. En este sentido, el crecimiento de los GTI's a partir de 1995 y hasta el año 2010 ha sido del orden del $41 \%$ para Accor International Hotels, $48.7 \%$ para Choice Hotels International, 45.6\% Intercontinental Hotels Group, 69.2\% Marriot International. La presencia de los GTI's en América Latina es importante, de tal forma que existen en 1,010 hoteles de los grupos que se han expandido a lo largo de mundo (Ver Tabla 2).

Tabla 2. Presencia de los GTI en el Mundo.

\begin{tabular}{|ccccc|}
\hline GTI & Principales Marcas & Hoteles & Hoteles & Hoteles \\
1995 & 2010 & ALyC \\
\hline $\begin{array}{c}\text { Accor } \\
\begin{array}{c}\text { International } \\
\text { Hotels }\end{array}\end{array}$ & $\begin{array}{c}\text { Ibis, Formule 1, Novotel, } \\
\text { Sofitel, Ticket Restaurant y } \\
\text { Ticket Alimentación } \\
\text { (serviciosprepagados) }\end{array}$ & 2378 & 4031 & 184 \\
\hline $\begin{array}{c}\text { Best Western } \\
\text { International }\end{array}$ & Best Western & 3462 & 4200 & 120 \\
\hline $\begin{array}{c}\text { Carlson } \\
\text { Hospitality } \\
\text { Worldwide }\end{array}$ & $\begin{array}{c}\text { Radisson, Regent, Country } \\
\text { Inn, T.G.I. Fridays, Pick Up } \\
\text { Stix, Carlson Wagonlit Travel }\end{array}$ & 383 & 548 & \\
\hline $\begin{array}{c}\text { Choice Hotels } \\
\text { International }\end{array}$ & $\begin{array}{c}\text { Clarion, Comfort Inn, Comfort } \\
\text { Suites, Econo Lodge, Quality, } \\
\text { Rodeway Inn }\end{array}$ & 2984 & 5827 & \\
\hline
\end{tabular}




\begin{tabular}{|c|c|c|c|c|}
\hline $\begin{array}{l}\text { Hilton Hotels } \\
\text { Corporation }\end{array}$ & $\begin{array}{l}\text { Hilton, Conrad, Embassy, } \\
\text { Hampton, Waldorf-Astoria }\end{array}$ & 219 & 514 & 47 \\
\hline $\begin{array}{l}\text { Hyatt Hotels } \\
\text { Corporation }\end{array}$ & Hyatt y Andaz & 172 & 207 & 13 \\
\hline $\begin{array}{l}\text { Intercontinental } \\
\text { Hotels Group }\end{array}$ & $\begin{array}{c}\text { Intercontinental Hotels, } \\
\text { Crowne Plaza, Holiday Inn, } \\
\text { Priority Club Rewards, Îndigo }\end{array}$ & 2275 & 4186 & 250 \\
\hline $\begin{array}{c}\text { Marriot } \\
\text { International }\end{array}$ & $\begin{array}{c}\text { Courtyard, J.W. Marriott, } \\
\text { Marriott Hotels and Resorts, } \\
\text { Ritz Carlton }\end{array}$ & 976 & 3178 & 59 \\
\hline $\begin{array}{l}\text { Starwood Hotels } \\
\text { \& Resorts } \\
\text { Worldwide }\end{array}$ & $\begin{array}{c}\text { Sheraton, Four Points, W } \\
\text { Hotels, Meridien, Westin, St. } \\
\text { Regis }\end{array}$ & 590 & 968 & 76 \\
\hline $\begin{array}{l}\text { Wyndham } \\
\text { Worldwide } \\
\text { Corporation }\end{array}$ & $\begin{array}{l}\text { Days Inn, Howard Johnson, } \\
\text { Ramada, RCI, Landal. }\end{array}$ & D.D. & 7043 & 123 \\
\hline Grupo Posadas & $\begin{array}{c}\text { Live Aqua, Fiesta Americana, } \\
\text { Explorean, Fista Inn, One } \\
\text { Hotels, }\end{array}$ & N.D. & 112 & 111 \\
\hline
\end{tabular}

Fuente: Elaboración propia con información de Clancy (1998), Palafox, Zizumbo y Arriaga (2010) y Palafox (2010).

Tal expansión del turismo como eje de acumulación requiere de un proceso que se resume de a continuación:

- Solicitud de préstamos de los países emergentes a los Organismos Internacionales (Banco Mundial [BM], Fondo Monetario Internacional [FMI], Organización para la Cooperación y el Desarrollo Económicos [OCDE], Banco Interamericano de Desarrollo [BID]) para la construcción de infraestructura de acceso y servicios.

- $\quad$ Reformas legales para la apropiación del territorio.

- Funcionalización del espacio para la instalación de la oferta turística complementaria (hoteles, restaurantes, plazas comerciales, entre otros).

- Arribo de los Grupos Turísticos Internacionales y expansión de las Global Commodity Chains (GCC).

- $\quad$ Eliminación de barreras arancelarias y precarización del empleo, así como de las condiciones laborales.

- Homogenización de la actividad, es decir, que en los destinos turísticos se encontrarán la misma oferta de bienes y servicios, como resultado del arribo de los GTI's y las Global Commodity Chains.

Los Organismos Internacionales direccionan la estrategia de libre mercado y las actividades económicas a desarrollar en los Estados subdesarrollados por medio de la otorgación de préstamos para el desarrollo del sector turístico, por ejemplo: el Banco de México e INFRATUR (Fondo de Promoción de Infraestructura Turística) solicitó un préstamo por 25 millones de dólares al Banco Interamericano 
de Desarrollo (BID) (INFRATUR, 1970) para el desarrollo de Cancún, lo anterior como resultado en las últimas décadas se detonaron reformas políticas de corte neoliberal a través de la racionalidad económica (Cornelius y Myhre, 1998), hoy en día, Cancún cuenta con 28,838 cuartos de hotel en 148 establecimientos, en suma a las 40,584 habitaciones de la Riviera Maya, también proyecto FONATUR (Fondo Nacional de Fomento al Turismo). En este sentido, el auge en la promoción del turismo principalmente por medio de la creación de los Centros Integralmente Planeados (CIP's) en el territorio mexicano, fue bajo el apoyo económico del Banco Mundial, Fondo Monetario Internacional y el Banco Interamericano de Desarrollo a través del Banco de México (Marín; 2012).

No obstante el apoyo recibido para la creación de infraestructura, el Estado modifica la Constitución, particularmente el Art. 27, para la apropiación y despojo del territorio con fines privatizadores y en consecuencia el desarrollo turístico se ha incrementado principalmente en zonas costeras. (Méndez y Garduño; 2013). Por otro lado, Gomezjara (1974) afirma que el despojo de tierras para fines turísticos se inició en 1928, un año después de la inauguración de la carretera México-Acapulco, cuando el Ayuntamiento donó terrenos del antiguo ejido colonial a quienes consideró como vecinos distinguidos. Así mismo, Nuevo Vallarta se consolida a través de la creación de Bahía de Banderas y la expropiación de tierra para la construcción de la infraestructura hotelera, campos de golf, entre otras actividades no tradicionales (Márquez, 2007). Por su parte, el CIP Loreto se convierte en punto de partida de la colonización de la península de Baja California (Davis, 1998).

Una de las consecuencias más relevantes de la expansión del turismo ha sido el encarecimiento de la tierra, al ser transformado en mercancía provoca la aparición de nuevos usos (Chavoya y García; 2010), a través de los Planes de Ordenamiento Urbano de los diversos municipios principalmente para aumentar los niveles en los límites de edificación (González; 2010) y designar los nuevos espacios para el turismo residencial. En este sentido, a inicios de la década de los setenta, FONATUR inició negociaciones con el BID a fin de que el CIP Loreto fuera financiado y a partir de ese instante se realiza el Plan Maestro de Desarrollo para la lotificación de la zona dotándola de 10,000 hectáreas para uso turístico (FONATUR; 1995 en De Sicilia; 2000). Del mismo modo sucedió en Cancún y Huatulco, en donde por medio de la Secretaría de Desarrollo Urbano y Ecología expropió a favor del FONATUR la utilidad pública del predio de 20,975 hectáreas para el desarrollo turístico (Diario Oficial de la Federación, 1984 en: López; 2011).

Tras la expropiación del territorio, la consecuencia se plasma en la llegada de trasnacionales, lo que generó una marcada dependencia económica y el turismo es prueba de ello. En suma a las facilidades otorgadas por el Estado posibilitan que el turismo se constituya como un eje de acumulación, este proceso fortalece la formación de Grupos Turísticos Internacionales y la consolidación e integración de nuevas Global Commodity Chains. Algunas comunidades receptoras han empleado este tipo de quehacer y han generado los Grupos Turísticos Locales (GTL's) (Palafox y Zizumbo; 2009; Palafox; 2010 y Palafox, Zizumbo y Madrigal; 
2011). Así, lejos de producir un desarrollo para las comunidades receptoras, la expansión del turismo bajo la lógica del capital "supone una nueva fuente de conflictos y contradicciones para las sociedades en las que se arraiga" (Blázquez y Cañada; 2011). Sin embargo, la expansión del turismo ha trascendido inclusive en países como Cuba, en donde los Grupos Turísticos Españoles han invadido la ínsula a través de 23,397 habitaciones en 58 hoteles. La cadena hotelera Sol Meliá tiene el $43.3 \%$ de los cuartos y el $39.6 \%$ de los establecimientos, el restante $56.7 \%$ es repartido en nueve cadenas, las cuales suman únicamente 25 hoteles (Riverón; 2010 en Navarro; 2011).

La exención de impuestos a favor del desarrollo turístico internacional se inserta en la dinámica que persigue la consolidación del turismo como eje de acumulación, por ejemplo en Marruecos las empresas hoteleras extranjeras tienen marcadas preferencias sobre las exoneraciones y reducciones, a saber: a) exención del impuesto de sociedades durante cinco años; b) exoneración del pago de derechos de registro para la constitución de sociedades, ampliación de capital o compra de terrenos; b) exoneración del IVA en mercancías importadas; d) exoneración del impuesto sobre el rendimiento de acciones o participaciones; e) exoneración de impuesto de patentes, tasa urbana impuesto sobre la renta por 15 años (Arguer; 2004). Del mismo modo, en Argentina se establece la exención del pago al impuesto por ingresos brutos para aquellos que empiecen la actividad hotelera en la provincia de Entre Ríos durante los cinco primeros años (Wallingre; 2008). Así mismo, la política turística pública de Colombia otorga los siguientes beneficios para el sector turístico del país, a saber: a) exención del impuesto sobre la renta por 15 años para nuevos establecimientos; b) exención del impuesto sobre la renta por 30 años a establecimientos de hospedaje que remodelen durante los próximos 15 años, y c) exención del impuesto sobre la renta por 20 años para servicios de ecoturismo (Toro; 2003). Lo opuesto ocurre en España, donde hay una exención de impuestos a empresarios locales y personas de la tercera edad, dicha política derivo en un empadronamiento del residente español y extranjeros con la finalidad de que el transporte público sea gratuito para ese sector de la población (Baños; 1999).

Finalmente, después de haber obtenidos las facilidades para que las empresas consoliden su presencia en el territorio, la expansión de las mismas es inminente, toda vez que cuentan con la apertura del mercado, la apropiación del espacio aunado a la funcionalización de la accesibilidad, sólo resta homogenizar la oferta, de esta manera, en todos los destinos turísticos fomentados por el Estado (principalmente) se hallarán las mismas empresas ofreciendo los mismos servicios por medio de la expansión y la estandarización (Ver Figura 1 y Tabla 3). 
Figura 1. Comportamiento de los GTI en América Latina y El Caribe 2010 - 2013.

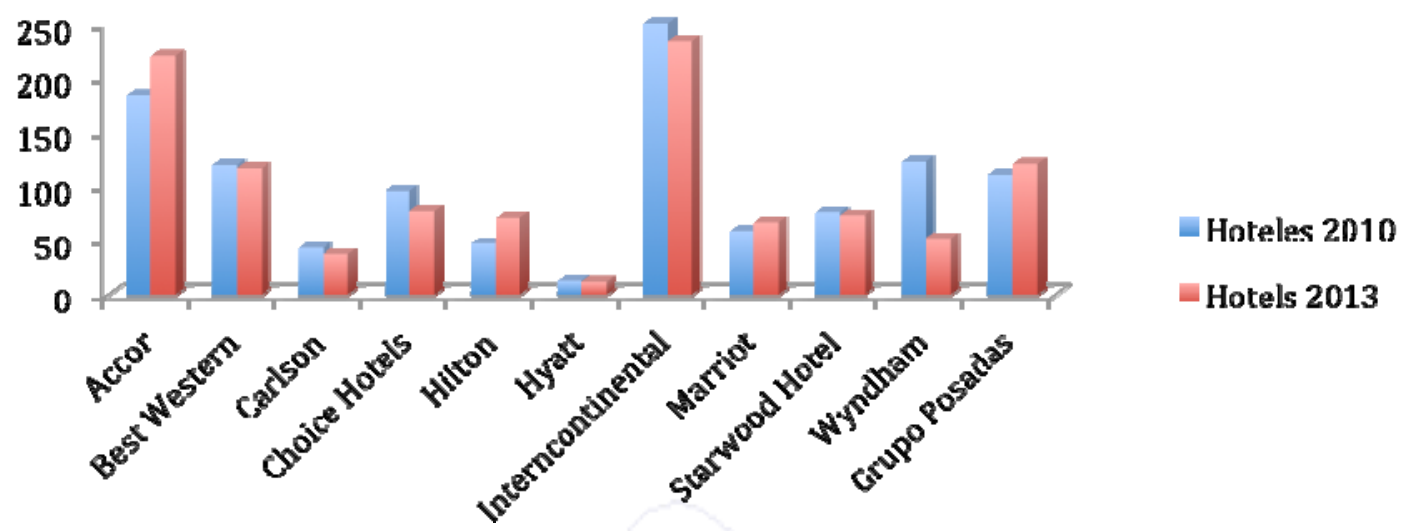

Fuente: Elaboración propia con información de Palafox, Zizumbo y Arriaga (2010) y Palafox (2010), www.accor.com, www.bestwestern.com, www.carlson.com, www.hhonors3.hilton.com, www.hyatt.com, www.ihg.com, www.marriot.com, www.cities.starwoodhotels.com, www.posadas.com

Tabla 3. Presencia de los GTI en América Latina y el Caribe

\begin{tabular}{|c|c|c|}
\hline GTI & País & $\begin{array}{l}\text { Hoteles } \\
2013\end{array}$ \\
\hline $\begin{array}{l}\text { Accor } \\
\text { International } \\
\text { Hotels }\end{array}$ & $\begin{array}{l}\text { Argentina, Brasil, Colombia, Chile, Ecuador, } \\
\text { Guatemala, México, Perú y Uruguay. }\end{array}$ & 221 \\
\hline $\begin{array}{l}\text { Best Western } \\
\text { International }\end{array}$ & $\begin{array}{c}\text { Antillas Holandesas, Argentina, Bahamas, Belice, } \\
\text { Bolivia, Brasil, Chile, Colombia, Costa Rica, } \\
\text { Ecuador, Guyana Francesa, Guatemala, Haití, } \\
\text { Islas Vírgenes, México, México, Nicaragua, } \\
\text { Panamá, Perú, Puerto Rico, Surinam, Uruguay y } \\
\text { Venezuela. }\end{array}$ & 117 \\
\hline $\begin{array}{l}\text { Carlson } \\
\text { Hospitality } \\
\text { Worldwide }\end{array}$ & $\begin{array}{c}\text { Aruba, Bahamas, Barbados, Belice, Bolivia, } \\
\text { Brasil, Chile, Colombia, Costa Rica, Ecuador, } \\
\text { Grenada, Guatemala, México, Panamá, Perú } \\
\text { Puerto Rico, Saint Martin y Uruguay. }\end{array}$ & 37 \\
\hline $\begin{array}{l}\text { Choice Hotels } \\
\text { International }\end{array}$ & $\begin{array}{l}\text { Bahamas, Brasil, Curazao, Guatemala, Islas } \\
\text { Caimán, Costa Rica, El Salador, Honduras, } \\
\text { México, Puerto Rico, República Dominicana. }\end{array}$ & 77 \\
\hline Hilton Hotels & $\begin{array}{c}\text { Argentina, Bahamas, Barbados, Brasil, Colombia, } \\
\text { Costa Rica, Chile, Ecuador, El Salvador, } \\
\text { Honduras, Jamaica, México, República } \\
\text { Dominicana, Perú, Puerto Rico, Trinidad y } \\
\text { Tobago, Uruguay y Venezuela . }\end{array}$ & 71 \\
\hline $\begin{array}{l}\text { Hyatt Hotels } \\
\text { Corporation }\end{array}$ & $\begin{array}{c}\text { Argentina, Brasil, Chile, Costa Rica, México, } \\
\text { Puerto Rico y Trinidad y Tobago }\end{array}$ & 12 \\
\hline
\end{tabular}




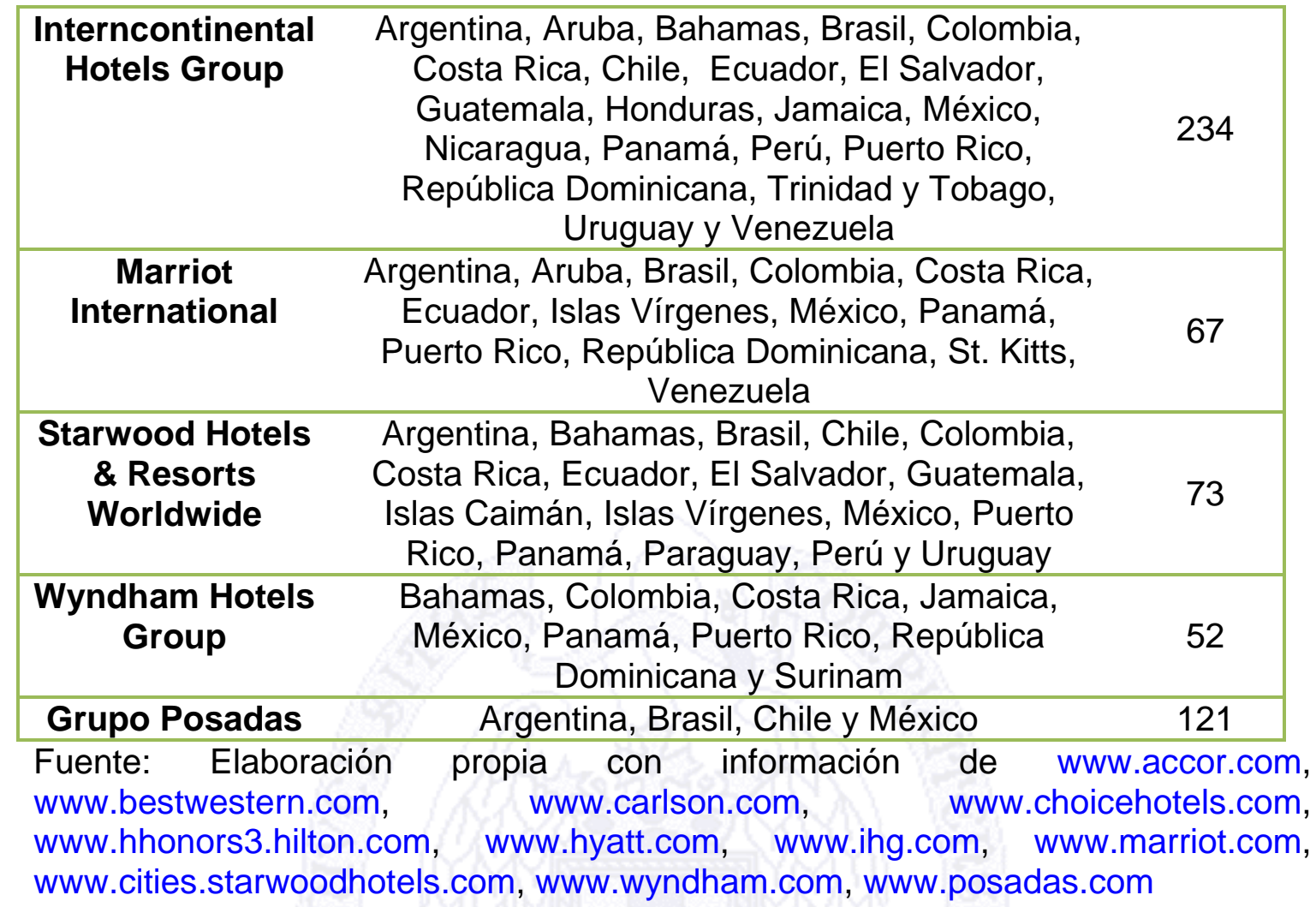

Los GTI's se han expandido de forma constante en América Latina y El Caribe, en este sentido Accor International Hotels cuenta con 221 establecimientos distribuidos en Argentina, Brasil, Colombia, Chile, Ecuador, Guatemala, México, Perú y Paraguay. Del mismo modo Best Western International suma 117 hoteles en 20 países de la región. Así mismo, Choice Hotels International cuenta con 77 establecimientos de hospedaje divididos en Bahamas, Brasil, Curazao, Guatemala, Islas Caimán, México, Costa Rica, El Salvador, Honduras, Puerto Rico, y la República Dominicana. Otro de los GTI que se ha expandido en la región Latinoamericana es International Hotels Group quien concentra 234 hoteles en 21 países. A pesar de la concentración del mercado por parte de los GTI's de capitales extranjeros, el Grupo Posadas tiene 121 establecimientos distribuidos principalmente en México y algunos hoteles en Brasil, Argentina y Chile. Por otro lado, Argentina y Colombia cuentan con la presencia del $72 \%$ de los GTI's, Puerto Rico con el $81 \%$, Brasil 90\% y México alberga el 100\% de los GTI's dentro de su territorio; la región concentra 1082 hoteles de los diez GTI's. 


\section{Conclusiones}

El turismo como eje de acumulación se ha establecido como plataforma para la reproducción del modo de producción, para ello la globalización económica por medio del libre mercado y la formación de cadenas productivas han contribuido a la formación de los Grupos Turísticos Internacionales que se han expandido en las economías emergentes, las cuales con la necesidad de generar empleo y crecimiento económico han optado por solicitar préstamos a los Organismos Internacionales para la construcción de infraestructura de acceso y servicios, elementos necesarios para la llegada de la inversión privada.

El Estado a través del turismo ha optado por el crecimiento económico en busca del desarrollo regional y nacional, sin embargo, las condiciones que ha establecido el mismo gobierno han provocado las empresas trasnacionales acaparen el mercado y exista una gran fuga de capitales y detrimento de las condiciones del empleo.

El turismo como eje de acumulación presenta como proceso: a) Solicitud de préstamos para la construcción de infraestructura; b) El Estado ha facilitado la llegada y consolidación de los Grupos Turísticos Internacionales y la apropiación del territorio; c) La funcionalización del espacio es necesaria para el arribo de turistas y el acceso a la oferta turística alterna al atractivo; d) Los Grupos Turísticos Internacionales se han expandido y diversificado a través de las Global Commodity Chains; e) El Estado ha exentado de cargas fiscales a la empresa privada lo que deriva en una nula captación de divisas para el desarrollo de la propia actividad; f) Los destinos turísticos han sido homogenizados por los Grupos Turísticos Internacionales con la finalidad de proveer al turista los espacios y productos que encuentran en sus países de origen.

Como resultado del turismo como eje de acumulación, los destinos turísticos han perdido toda identidad que pueda diferenciarlos, esta estandarización provoca el estancamiento de la actividad a pesar de la diversidad de paisajes con que cuentan los destinos turísticos. 


\section{Literatura citada}

a) (1969) FRANK, Andre Gunder, Capitalism and Underdevelopment in Latin America, Monthly Review Press, New York.

b) (1970) INFRATUR, Proyecto de desarrollo turístico de Cancún. Resúmen de la propuesta de préstamo a Nacional Financiera, S.A., Fondo de Promoción de Infraestructura Turística, Distrito Federal.

c) (1974) GOMEZJARA, Francisco, "Acapulco: despojo y turismo". Problemas del desarrollo, Año V, ISSN 0301-7036, pp. 126 - 147, Distrito Federal.

d) (1991) BRITTON, Stephen, Tourism, capital and place: towards a critical geography of tourism. In: Environment and Planning D: Society and Space, No. 9, pp. 451 - 478, ISSN 0263-7758, London.

e) (1995) FONATUR, Loreto - Nopoló - Puerto Escondido. Centro Integralmente Planeado por el Fondo Nacional de Fomento al Turismo, Fondo Nacional de Fomento al Turismo, Distrito Federal.

f) (1998) CLANCY, Michael, "Commodity chains, services and development: Theory and preliminary evidence from the tourism industry". Review of International Political Economy, No. 5, ISSN 0969-2290, pp. 122 - 148, New York.

g) (1998) CORNELIUS, Wayne. A. y MYHRE, David, The Transformation of Rural Mexico: Reforming the Ejido Sector. San Diego/La Jolla: Center for USMexican Studies, University of California. San Diego.

h) (1998) DAVIS, Estela, El alojamiento en Baja California Sur, Coordinación Estatal de Turismo - Colegio de Bachilleres del Estado de Baja California Sur, La Paz.

i) (1999) BAÑOS CASTIÑEIRA, Carlos Javier, "Modelos turísticos locales. Análisis comparado de dos destinos de la Costa Blanca". Investigaciones Geográficas, No. 21, ISSN 0213-4691, pp. 35 - 57, Alicante.

j) (1999) MARX, Karl, El Capital. Crítica a la economía política, Fondo de Cultura Económica, Distrito Federal.

k) (2000) DE SICILIA MUÑOZ, R. A. "El corredor turístico Loreto - Nopoló Puerto Escondido, Baja California Sur, en el contexto de los Centros Integralmente Planeados". Cuadernos de Turismo, No. 5, ISSN 1139-7861, pp. 53 - 68, Murcia.

I) (2002) WILLIAMS, Mariama, Tourism Liberalization, Gender and the GATS, International Gender and Trade Network, Rio de Janeiro.

m) (2003) BRENNAN, Teresa, Globalization and its terrors. Routledge, London.

n) (2003) HILLALI, Mimoun, Le tourisme international vu du Sud, Quebec, Presses de l' Université du Québec, Quebec.

o) (2003) REID, Dondald G., Tourism, Globalization \& Development: Responsible Tourism Planning, Pluto Press, London.

p) (2003) TORO, Gustavo, "La política pública de turismo en Colombia", Turismo y sociedad, Vol. 3, ISSN 0120-7555, pp. 9 - 15, Bogotá.

q) (2004) ARGUER, Óscar. "El sector turístico - hotelero en Marruecos". Información Comercial Española - Revista de Economía, No. 189, ISSN 0019977X, pp. 145 - 156, Madrid.

r) (2004) LANFANT, Marie Françoise, "L'appe à I éthique et la reference universaliste dans la doctrine officielle du turisme international". Revue Tiers Monde; Vol. 45, No. 178, ISSN 1293-8882pp. 365 - 386, Paris. 
s) (2006) MOSEDALE, Jan, "Tourism commodity chains: Market entry and its effects on St. Lucia", Current Issues in Tourism, No. 9, ISSN 1368-3500, pp. 436 458, London.

t) (2006) ZHAO, Weibing \& LI, Xingqun, "Globalization of Tourism and Third World Tourism Development", Chinese Geographical Science, Vol. 16, No. 3, ISSN 1002-0063, pp. 203 - 210, Changchun.

u) (2007) DUTERME, Bernard, "Turismo hoy: ganadores y perdedores". In: Bernard Duterme. (Coord.). Turismo hoy: ganadores y perdedores: alternativas meridionales, Editorial a la Izquierda, Madrid, pp. 7 - 27.

v) (2007) MÁRQUEZ GONZÁLEZ, Antonio Romualdo, La expansión del turismo costero en Bahía de Banderas, Nayarit: un análisis de deterioro ambiental, Tesis de Doctorado en Geografía, Universidad Nacional Autónoma de México, Distrito Federal.

w) (2008) WALLINGRE, Noemí, "Evolución del desarrollo del turismo en la provincia de Entre Ríos, Argentina", Tiempo de Gestión, No. 5, ISSN 1850-7255, pp. 13 - 34, Entre Ríos.

x) (2009) BIANCHI, Raoul. V., "Tourism, capitalism and Marxist political economy". In: Jan Mosedale. (Ed.), Political Economy of Tourism: A Critical Perspective, Routledge, London, pp. $17-37$.

y) (2009) MOSEDALE, Jan, "Re-introducing tourism to political economy", In: Jan Mosedale (Ed.), Political Economy of Tourism: A Critical Perspective, Routledge, London, pp. $1-14$.

z) (2009) WEBSTER, Craig, IVANOV, Stanislav \& ILLUM, Steven, "The paradigms of political economy and tourism policy". Jan Mosedale (Ed.), Political Economy of Tourism: A Critical Perspective, Routledge, London, pp. 55 - 73.

aa) (2010) CHAVOYA GAMA, Jorge Ignacio y GARCÍA GALVÁN, Joel, "Ciudades para el turismo: La costa del pacífico mexicano; memoria, imagen y fantasía". Topofilia, Vol. II, No. 1, ISSN en Trámite, Hermosillo. http://topofilia.net/chavoya-garc\%C3\%ADa.pdf

bb) (2010) GONZÁLEZ ROMERO, Daniel, "Ciudad virtual, turismo, imaginario y realidad social en Puerto Vallarta: luces, sombras, abordajes, motines y naufragios". Architecture, City and Environment (ACE), Año 4, No. 12, ISSN 18864805, pp. 101 - 113, Barcelona.

cc) (2010) MARX, Karl, El capital: antología, Alianza Editorial, Madrid.

dd) (2010) PALAFOX MUÑOZ, Alejandro, "Treinta años de transformaciones en Cozumel: El turismo como eje de acumulación", en: Revista de Economía, Sociedad, Turismo y Medio Ambiente (RESTMA), No.11, ISSN 1698-8280, pp. 105 - 122, Oviedo.

ee) (2010) PALAFOX MUÑOZ, Alejandro, ZIZUMBO VILLARREAL, Lilia, y ARRIAGA ÁLVAREZ, Emilio, "El turismo como eje de acumulación: el caso del sector hotelero en México", Multiciencias, Vol. 10, No. 2, ISSN 0798-2259, pp. 193 - 202, Maracaibo.

ff) (2010) RIVERÓN ABREU, Claudia, La participación española en la hotelería cubana. Un contribución a su estudio, Tesis de Licenciatura en Turismo, Universidad de La Habana, La Habana.

gg) (2011) BUADES, Joan, "Geopolítica, neoliberalismo y turismo en los países catalanes". Alba Sud; No. 10, pp. 1 - 18, Catalunya. 
hh) (2011) LÓPEZ GUEVARA. Víctor Manuel, "La reorientación del ciclo de vida del área turística: el caso de Bahías de Huatulco, Oaxaca (México)". Investigaciones Turísticas, No.1, ISSN 0213-4691, pp. 107 - 121, Alicante

ii) (2011) NAVARRO JURADO, Enrique, "Turismo en Cuba: características e importancia de las empresas trasnacionales turísticas en su desarrollo". In: Macià Blázquez y Ernest Cañada (Coords.), Turismo Placebo, Editorial Enlace, pp. 75 100, Managua.

jj) (2011) PALAFOX MUÑOZ, Alejandro, ZIZUMBO VILLARREAL, Lilia y MADRIGAL URIBE, Delfino, "Apropiación, funcionalización y homogenización del espacio para el desarrollo turístico de Quintana Roo, México", Caderno Virtual de Turismo, Vol. 11, No. 2, ISSN 1677-6976, pp. 282 - 293, o de Janeiro.

kk) (2012) MARÍN GUARDADO, Gustavo, "Los tristes trópicos del turismo en México: industria, reflexividad y otras ficciones". In: Gustavo Marín Guardado, Ana García de Fuentes y Magaly Daltabuit Godás (Coords.), Turismo, globalización y sociedades locales en la península de Yucatán, México, Colección Pasos Edita, Tenerife, pp. $17-44$.

II) (2012) OMT. UNWTO Hihglights 2012, Madrid, Organización Mundial del Turismo.

mm) (24 de abril de 2013) MÉNDEZ, Enrique y GARDUÑO, Roberto, "Diputados aprueban reforma que permite a extranjeros comprar inmuebles en playas". In: La Jornada, http://www.jornada.unam.mx/2013/04/24/politica/020n1pol, [24/04/2013].

nn) (24 de mayo de 1984) DIARIO OFICIAL DE LA FEDERACIÓN, Decreto expropiatorio de la comunidad denominada Santa María Huatulco, municipio de Santa María Huatulco, estado de Oaxaca. Secretaría de Gobernación. Distrito Federal. 